\title{
Reentrant Phase Separation in Microemulsions
}

\author{
R. Menes and S. A. Safran \\ Department of Materials and Interfaces, Weizmann Institute of Science, Rehovot 76100, Israel \\ R. Strey \\ Max-Planck-Institut für Biophysikalische Chemie, Postfach 2841, 37018 Göttingen, Germany
}

(Received 9 August 1994)

\begin{abstract}
We propose a mechanism for reentrant phase separation in globular microemulsions based on the combined effects of a shape transition and attractive interactions. Long cylindrical globules can phase separate at relatively low interglobular attractions. A transformation from elongated globules to compact spherical drops alters the balance between the entropy and effective interglobule interactions, leading to the remixing of the globular system. Our theory qualitatively explains the closed-loop coexistence regions seen in recent experiments on nonionic surfactant microemulsions.
\end{abstract}

PACS numbers: 64.70.Ja, 64.75.+g, 82.70.-y

Recent experiments on microemulsions (ME) containing nonionic surfactants exhibit closed loops where two ME phases coexist [1]; the loops show both upper and lower critical points in the isothermal ternary phase diagrams [2], and appear on both the water rich and the oil rich corners of the diagram, depending on the temperature of the system. The temperature also affects the size of the coexistence region. NMR experiments [3] suggest a shape transformation of the globular ME in the region of the closed loops.

Reentrant phase separation and closed-loop coexistence regions require, in addition to a basic phase separating mechanism, an additional degree of freedom that can counteract this mechanism and cause the reentrant behavior. Examples of systems exhibiting this phenomenon are binary mixtures where hydrogen bonding dominates the orientational entropy of the molecules at low temperatures [4]. In some cases, the bonding is strongest between unlike molecules, inducing remixing at low temperatures. The mixing at high temperatures arises from the translational entropy, while the remixing at low temperatures is due to the additional, orientational, degrees of freedom. Three-component ME [5], which are liquid mixtures of water, oil, and surfactant, can form droplets or other globular shapes which can show phase separation due to the competition between attractions and entropy. However, in addition, this self-assembling system also has degrees of freedom related to the shapes of the globules, which can cause remixing.

We propose a mechanism based on the combined effects of attractive interactions and a shape transition of the ME droplets which explains the existence of such reentrant phases and the appearance of both upper and lower critical points on closed-loop diagrams. We show that the closed loops in the composition plane are produced at constant temperature. The temperature affects the size and shape of the isothermal coexistence regions through the interfacial parameters. We find that as a function of the ratio $r$ of internal phase (water or oil) and surfactant volume fractions, $\phi_{i} / \phi_{s}$, the system undergoes an isothermal transition from thin, elongated cylindrical globules to more compact spheres [6]. Initially, the system of very thin cylinders is homogeneous. However, as $r$ is increased the cylinders become thicker, thus increasing the effective interglobule attractions, and inducing phase separation into high and low density states. Increasing $r$ further, the ME consists of coexisting cylinders and spheres [7]. The smaller spherical objects which become more numerous as $r$ increases, have higher translational entropy which restabilizes the homogeneous phase and causes remixing of the system. Our mechanism is related to the existence of a finite interval of attraction strengths where long cylinders will phase separate, while compact spheres stay homogeneous. In standard models of phase separation of long polymers, one finds polymer-solvent phase separation at attractions which are up to 4 times weaker than the attraction needed to phase separate a system of monomers [8].

We treat the ME within the interfacial model [9], which assumes that the properties of the three-component system are determined by the physics of the surfactant-saturated interface between the water and oil. This approach is appropriate to "good" surfactants which are insoluble in both the water and oil. Our model takes into account, besides the interfacial bending energy $[10,11]$ and the entropy of the globules, an attractive interaction, such as van der Waals attraction [12].

In general, the ME will include a distribution of shapes and sizes of globules which is difficult to treat theoretically. In order to simplify the theoretical treatment we consider a system composed of cylinders of varying lengths but with uniform radii. This choice is justified in systems with large bending moduli, where the bending energy dictates the equilibrium uniform radii, but the cylinder length is determined by a weaker, entropic contribution. In this model, for a certain range of parameters, the cylinders coexist with monodisperse spheres. 
Long enough cylinders are flexible and can be treated as a system of self-assembled (living) polymers [6]. The cylinders have been shown to have a persistence length which has the form $\xi \sim K r_{c} / T$ [6], where $K$ is the bending modulus of the surfactant interface and $r_{c}$ is the cylinder radius. $\xi$ is identified with the "monomer" size, thus completing the polymeric analogy.

We treat the hard core repulsion and short range attraction between the various types of globules by considering a lattice model. The lattice must accommodate both the long cylinders and the compact spheres. The natural lattice size for the cylinders is the above mentioned monomer size, while the optimal lattice size for the spheres is related to their volume. As will be shown below the cylinder and sphere radii, $r_{c}$ and $r_{s}$, are of the same order of magnitude. Therefore, we use a mean cell size which is correct in the two limits of pure cylinders and pure spheres and smoothly interpolates between these two limits. The volume, $V_{0}$, of this cell is

$$
V_{0}=\left(X_{c} \pi r_{c}^{2} \xi+X_{s} \frac{4}{3} \pi r_{s}^{3}\right) / \phi,
$$

where $r_{s}$ is the sphere radius, $X_{s}$ is the sphere volume fraction, and $X_{c}=\sum_{m} X_{m}$ is the total cylinder volume fraction given by the infinite sum over volume fractions of cylinders with different lengths, where the length is measured in terms of the number, $m$, of persistence lengths. $\phi=X_{c}+X_{s}=\phi_{i}+\frac{1}{2} \phi_{s}$ is the total globule volume fraction.

The bending energy of a single globule is given by $[10,11]$

$$
\begin{aligned}
E_{B}= & \frac{K}{2} \int\left(\frac{1}{R_{1}}+\frac{1}{R_{2}}-\frac{2}{\rho_{0}}\right)^{2} d S \\
& +\frac{\bar{K}}{2} \int\left(\frac{1}{R_{1}}-\frac{1}{R_{2}}\right)^{2} d S,
\end{aligned}
$$

where $K$ and $\bar{K}$ are the bending moduli, $\rho_{0}$ is the spontaneous radius of curvature of the interface, $R_{1}$ and $R_{2}$ are the local radii of curvature, and $d S$ is the surface area element [13].

The contribution to the free energy per unit volume from the entropy of mixing of the globules is approximately [14] given by the Flory-type expression [8],

$f_{e}=\frac{T}{V_{0}}\left[\sum_{m} \frac{X_{m}}{m} \ln \frac{X_{m}}{m}+X_{s} \ln X_{s}+(1-\phi) \ln (1-\phi)\right]$.

The interaction between globules is also treated in the Flory approximation. Using a contact energy, $\chi$, the interaction per unit volume is given by $\tilde{\chi} \phi^{2}$, where $\tilde{\chi}=$ $\chi / V_{0}$. The contact energy $\chi$ depends on the lattice size; within the Derejaguin approximation [11], $\chi \propto V_{0}^{1 / 3}$.

Finally, the grand potential per unit volume has the form

$g=\sum_{m} n_{m} E_{B}(m)+n_{s} E_{B}(s)+f_{e}-\phi^{2} \tilde{\chi}+\mu \phi_{i}+\lambda \phi_{s}$.
Here $E_{B}(m)$ and $E_{B}(s)$ are the cylinder and sphere bending energy calculated from Eq. (1) using $R_{1}=r_{c}, R_{2}=\infty$ for the cylinders, and $R_{1}=R_{2}=r_{s}$ for the spheres. $n_{m}$ is the number of cylinders with $m$ persistence lengths per unit volume, and $n_{s}$ is the number of spheres per unit volume. The number densities are related to the volume fractions through $X_{m}=n_{m} V_{c}(m)$ and $X_{s}=n_{s} V_{s}$, where $V_{c}(m)$ is the volume of a cylinder with $m$ persistence lengths and $V_{s}$ is the sphere volume. $\mu$ and $\lambda$ are the internal phase and surfactant chemical potentials which constrain their respective volume fractions.

To determine the coexistence region, one first minimizes $g$ with respect to the radii $r_{s}$ and $r_{c}$, and then with respect to $\phi_{i}$ and $\phi_{s}$. This turns out to be rather complicated if we take into account all contributions to the energy. In order to simplify the calculation we work in the limit of the large bending modulus, $K \gg k_{B} T, \chi$. This allows us to estimate $r_{s}$ and $r_{c}$ by taking into account only the bending energy, neglecting all other contributions to the free energy. We then use the fact that the average length of the cylinders grows exponentially with $K$ [6], and calculate the equilibrium values for $r_{s}$ and $r_{c}$ in the limit of a system of infinitely long cylinders. This approximation is good only in the limit of large $K$ and small $\bar{K} / K$, where the cylinders are indeed long [15]. In the region of pure cylinders, the radius is dictated by the composition constraints [6] and is given by $r_{c}=2 \phi \delta / \phi_{s}$, where $\delta$ is the surfactant film thickness. In the region where cylinders and spheres coexist the radii are found by the minimization process. To first order in $\bar{K} / K$ they are $r_{c}=\frac{1}{2} \rho_{0}\left(1-\frac{1}{2} \bar{K} / K\right)$ and $r_{s}=\rho_{0}(1-2 \bar{K} / K)$.

The cylinder length distribution is found by minimizing Eq. (3) with respect to $X_{m}$. We find that the volume fraction of cylinders of length $m$ is given by

$$
X_{m}=m t^{m} e^{-E_{f}(R)-1},
$$

where $E_{f}(R)$ is the bending energy of the end cap needed to close a finite cylinder, calculated from Eq. (1), and $t$ is given by the solution to the quadratic equation [16] given by the constraints

$$
t^{2}-2 t\left(1+\frac{e^{-E_{f}(R)-1}}{\phi} g(R)\right)+1 \simeq 0 .
$$

Here $g(R)$ is a smooth function of the dimensionless parameter $R=3\left(\phi / \phi_{s}\right) \delta / \rho_{0}$ and $g(R)=R / 6$ for small $R$ where the ME is essentially cylindrical, while for $R \approx 1$, $g(R) \sim(1-R)^{-1}$. We must choose the "minus" solution of this quadratic equation in order to get $t<1$. This is necessary in order to get a converging distribution, $X_{m}$. The resulting average cylinder length is exponentially dependent on the bending moduli, $K$ and $\bar{K}$. An increase in $K$ increases this average, while $\bar{K}>0$ decreases the average. The sphere volume fraction (in the coexistence region) is given by the conservation law

$$
X_{s}=\phi-\sum_{m} X_{m}
$$




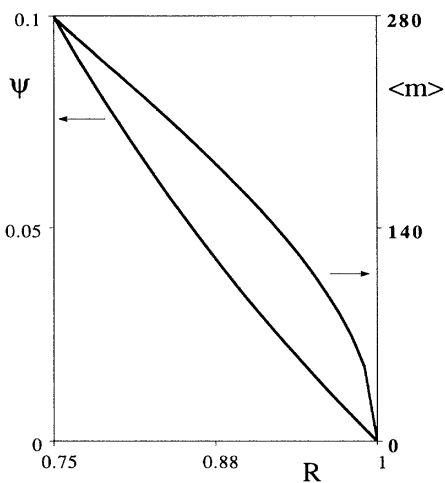

FIG. 1. Fraction of cylindrical globules, $\psi$, and average cylinder length, $\langle m\rangle$, as a function of $R=3 \phi \delta / \phi_{s} \rho_{0}$, in a system with $K=2 k_{B} T, \bar{K}=0$, and $\phi=0.1$. Note that $R=$ 0.75 is the transition point where cylinders coexist with spheres.

Figure 1 shows how the total number of cylinders and their average length decrease as $R$ increases beyond the point where spheres coexist with the cylinders. This trend is responsible for the reentrance of the single, uniform phase.

Using Eq. (4) we have an explicit form for the free energy. One notes that the two volume fractions, $\phi_{i}$ and $\phi_{s}$, enter only through $\phi$ and $R$. In the limit of large $K$, the dominant $R$ dependence is through $e^{-E_{f}(R)}$. In this case $R$ can be treated as an external parameter since its fluctuations are negligible and we are left with a problem with one concentration, $\phi$ [17].

In Fig. 2 we show partial isothermal phase diagrams in the region of small volume fractions. The plots show the spinodal curves (given approximately by the locus of $\left.\partial^{2} g / \partial \phi^{2}=0[17]\right)$, which provide a limit on the stability

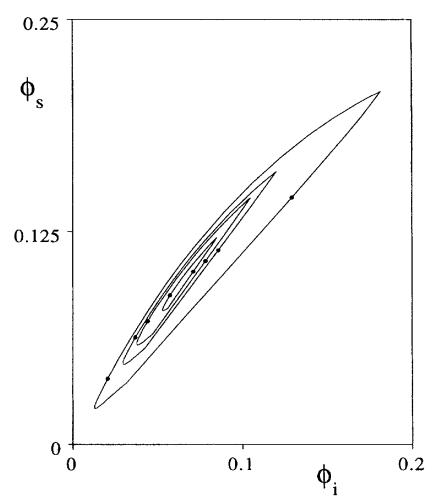

FIG. 2. Spinodal curves in the composition phase diagram of a system with $K=2 k_{B} T, \bar{K}=0$, and $\chi=0.15 k_{B} T$. The innermost loop corresponds to the smallest radius of curvature, $\rho_{0}=4.8 \delta$, and the outermost curve corresponds to the highest radius of curvature, $\rho_{0}=5.5 \delta$. The spinodal regions become progressively larger as $\rho_{0}$ is increased. The black circles indicate the upper and lower critical points. of the single phase of this model. The figures also show the development of the closed loops as the spontaneous curvature is decreased. In systems with large $K$, the upper parts of the closed loops are crowded together, although they do not actually touch. This tendency is less pronounced for smaller $K$.

To relate our results to the experiments, one must identify the parameters most sensitive to the temperature, which is the control parameter in experiment. The parameters $K / T$ and $\bar{K} / T$ are not changed much by the temperature changes induced in the laboratory $\left(30-40{ }^{\circ} \mathrm{C}\right.$, at most). However, the spontaneous radius of curvature, $\rho_{0}$, is strongly influenced by these temperature changes $[18,19]$. Within the relevant experimental temperature range, $\rho_{0}$ can even change sign, and the ME inverts [20]. We therefore interpret the different phase diagrams at different temperatures as systems with varying spontaneous curvatures. In the experiments the isothermal closed loops become larger as the spontaneous radius of curvature increases [21], in qualitative agreement with our model, as is clear from Fig. 2. The spontaneous curvature affects both the upper part of the loop (through the average cylinder length) and the lower part of the loop (through the curvature dependent effective attractions). The model is only sensitive to the structure and size of the ME, and not to the exact form of the attractions.

The model also reproduces the experimental trend where the reentrant single phase region, at large values of $R$, vanishes when the closed loop intersects the region of emulsification failure $[19,22]$. In the emulsification failure region the $\mathrm{ME}$ coexists with a nearly pure phase of the internal component $[3,23]$ which is rejected once the globule size is equal to the spontaneous radius of curvature. The width of the gap depends on temperature or, as we interpret, on the spontaneous curvature. Figure 3 demonstrates how this gap narrows as the spontaneous radius of curvature is increased in our model. This is in accordance with the interpretation of the experimental results $[1,22]$.

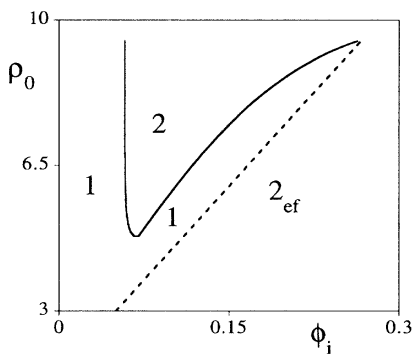

FIG. 3. Spinodal curve (solid line) and emulsification failure line (dashed line) as a function of the internal phase concentration, $\phi_{i}$, and the spontaneous radius of curvature, $\rho_{0}$, measured in units of the film thickness, $\delta$. The 2 indicates the two phase region calculated, while $2_{\text {ef }}$ describes the emulsification failure region where a spherical ME coexists with additional, rejected, internal phase. The curves are calculated for $\phi_{s}=0.1$. 
In experiment, the narrowing of the gap with temperature and the intersection of these two coexistence regions leads to the important three phase coexistence region. Our model indicates how this narrowing comes about [24].

At present, experiments indicate that the ME structure in the coexistence region is elongated, although the exact shapes have not been measured. Our theory is most applicable in the region of large $K$ and small $\bar{K} / K$ where we have considered a system of long, closed cylinders. Even if the ME structure is more complicated, we still expect both the entropy and interactions to depend (qualitatively) on $R$ in the same way as in our theory. As $R$ increases we expect the system to transform from thin elongated objects (where the attractions are too weak to cause phase separation), to thicker elongated objects (where the attractions induce demixing), to small compact droplets (where the entropy is strong enough to cause remixing). A system with less idealized shapes should still preserve these features essential to reentrant behavior.

The authors thank E. Kaler for useful discussions and acknowledge the support of the Israel Science Foundation administered by the Israel Academy of Sciences and Humanities.

[1] M. Kahlweit, R. Strey, and G. Busse, J. Phys. Chem. 94, 3881 (1990).

[2] Phase diagrams measured at constant temperature with respect to the relative compositions of the three components.

[3] M.S. Laver, U. Olsson, H. Wennerström, and R. Strey, J. Phys. II (France) 4, 515 (1994).

[4] J. S. Walker and C. A. Vause, Sci. Am. 256, No. 5, 90 (1987).

[5] See, for example, Structure and Dynamics of Strongly Interacting Colloids and Supramolecular Aggregates in Solution, edited by Sow-Hsin Chen, John S. Huang, and Piero Tartaglia, NATO ASI Ser. C, Vol. 369 (Kluwer Academic Publishers, Dordrecht, 1992), Chap. II.

[6] S. A. Safran, L. A. Turkevich, and P. Pincus, J. Phys. (Paris), Lett. 45, L-69 (1984).

[7] S. A. Safran, Phys. Rev. A 43, 2903 (1991).

[8] Paul J. Flory, Principles of Polymer Chemistry (Cornell University Press, Ithaca, NY, 1981), Chap. XII.

[9] G. Gompper and M. Schick, Phase Transitions and Critical Phenomena, edited by C. Domb and J. Lebovitz (Academic Press, London, 1994), Vol. 16.

[10] W. Helfrich, Z. Naturforsch. 28c, 693 (1973).
[11] Samuel A. Safran, Statistical Thermodynamics of Surfaces, Interfaces, and Membranes (Addison-Wesley Publishing Company, Reading, MA, 1994).

[12] Jacob N. Israelachvili, Intermolecular and Surface Forces (Academic Press, New York, 1992).

[13] The bending moduli and spontaneous radius of curvature are related to the standard Helfrich parameters of Ref. [10] through: $K=\kappa+\bar{\kappa} / 2, \bar{K}=-\bar{\kappa} / 2$ and $\rho_{0}=C_{0}^{-1}(1+$ $\bar{\kappa} / 2 \kappa)$.

[14] Since the cell size is not optimal either for the cylinders or the spheres, the mixing entropy is not exact within the lattice model. However, the error is not large since the cell size is adjusted according to the relative volume fractions.

[15] The cylindrical phase is stable in the region $0 \leq \bar{K} / K \leq$ 0.3 . For larger $\bar{K} / K$ the bending energy is minimized by spheres only.

[16] In this approximate equation we have neglected terms involving the small parameter $e^{-E_{f}-1} / K$. The full equation was used to obtain the results shown in Figs. 1-3.

[17] Within the framework of our initial approximation as to the monodispersity of radii, we also assume that two coexisting phases will have the same shape distribution, which is the physical meaning of working at constant $R$. This is included in the assumption that $K$ is large enough to dominate the shape minimization, while the densities of the coexisting phases are determined by the entropy and interactions only. Note that for $K>2$ the stability regions calculated for the one variable problem and for the two variable problem are essentially the same. As $K$ decreases the instability regions are less congruent, but the qualitative behavior is similar.

[18] M. E. Cates, D. Andelman, S. A. Safran, and D. Roux, Langmuir 4, 802 (1988); R. Strey, Colloid Polym. Sci. (to be published); David Anderson, Hàkan Wennerström, and Ulf Olsson, J. Phys. Chem. 93, 4243 (1989).

[19] Ulf Olsson and Hảkan Wennerström, Adv. Colloid Interface Sci. 49, 113 (1994).

[20] One sees in experiment that the system is very symmetric in the sense that reentrant phenomena are seen both for positive spontaneous curvature (on the water rich side of the diagram) and negative spontaneous curvature (on the oil rich side of the diagram).

[21] R. Strey and T. Sottmann (unpublished).

[22] M. Kahlweit, R. Strey, and G. Busse, Phys. Rev. E 47, 4197 (1993).

[23] S. A. Safran and L.A. Turkevich, Phys. Rev. Lett. 50, 1930 (1983).

[24] R. Menes, S. A. Safran, and R. Strey (to be published). 